year of diagnosis were associated with mortality in the multivariate regression model.

Conclusion This large population level TB study identifies an alarming trend of patients dying within months of starting treatment. These early deaths could be due to late diagnosis and multidrug-resistance. The study warrants further investigation to go beyond already established indicators which remained constant (including HIV co-infection), to explore host, disease or health system related factors that may explain the observed trend.

\section{PO 8418 NEW MALARIA EPIDEMIOLOGY IN COASTAL LAGOON OF BENIN: PLASMODIUM INFECTION IN ANOPHELES MELAS}

Claudiane Adigbonon*, Benoît S Assogba, Luc S Djogbenou. University of Abomey-Calavi, Cotonou, Benin

\subsection{6/bmjgh-2019-EDC.90}

Introduction Malaria is a worldwide disease affecting many people particularly in the tropical and sub-tropical areas. It is caused by Plasmodium parasites and essentially transmitted by female mosquitoes belonging to the Anopheles genus. Our understanding of the infectivity of these vectors to Plasmodium is necessary to design sustainable strategies for their control. This aspect remains unknown in the coastal and lagoon area of Benin where Anopheles melas and Anopheles coluzzii are sympatric. This study aims to investigate the infectivity of these two vectors to Plasmodium to understand their role in malaria transmission in southern Benin.

Methods Insecticide spray catch technique was used to collect females in 80 houses randomly selected in our study site. Three hundred and twenty (320) females were identified using PCR-species technique, Plasmodium infection was determined by the TaqMan method during the dry season. This assay detects all four malaria-causing species and discriminates Plasmodium falciparum from Plasmodium ovale, Plasmodium vivax and Plasmodium malariae (OVM).

Results During the dry season, the sporozoïte rates were $0.2 \%$ and $0.3 \%$ for Anopheles melas and Anopheles coluzzii, respectively. However, we observed that positivity to the OVM (one of Plasmodium ovale, Plasmodium vivax and Plasmodium malariae species) was significantly higher in Anopheles melas (95\%) than in Anopheles coluzzii (33.33\%) (Chi-sq=15 857, $\mathrm{df}=1, \mathrm{p}<0.001)$. These results indicated that Anopheles melas is more infected by one of the species Plasmodium ovale, Plamodium vivax and Plasmodium malariae than by Plasmodium falciparum, contrarily to Anopheles coluzzii.

Conclusion These findings reinforce the debate on the role of Anopheles melas in malaria transmission in coastal lagoon areas of Benin.

\section{PO 8419 SPATIO-TEMPORAL MAPPING OF ASYMPTOMATIC AND CLINICAL MALARIA INFECTIONS REVEALS FOCI OF MALARIA TRANSMISSION FOR TARGETED CONTROL INTERVENTIONS}

\footnotetext{
${ }^{1}$ Makhtar Niang* ${ }^{2}$ Cheikh Talla, ${ }^{3}$ Nafissatou Diagne, ${ }^{4}$ Fatoutama Diene-Sarr, ${ }^{3}$ Cheikh Sokhna. ${ }^{1}$ Immunology Unit, Institut Pasteur de Dakar, Senegal; ${ }^{2}$ Epidemiology Unit, Institut Pasteur of Dakar, Senegal; ${ }^{3}$ Institute for Research and Development, Dakar, Senegal; ${ }^{4}$ Epidemiology Unit, Institut Pasteur de Dakar, Senegal
}

10.1136/bmjgh-2019-EDC.91
Background The global decline of malaria incidence over the past decade has led to the thought that elimination could be achieved. This has resulted in an increased interest to design strategies to target the hidden reservoir of asymptomatic infections among populations and interrupt on-going residual local malaria transmission. This study explored the reservoir of asymptomatic Plasmodium infections and its relationship with subsequent clinical malaria infections in low-transmission areas in Senegal.

Methods Cross-sectional surveys were carried out in 2013, 2014, 2015, and 2016 and combined with longitudinal follow-up to determine and geolocalise both asymptomatic and clinical malaria episodes in Dielmo and Ndiop, Senegal. The prevalence of asymptomatic Plasmodium carriage in the community was investigated by real-time PCR while clinical malaria attacks were identified at health facilities during the transmission season. All households were georeferenced to spatially map asymptomatic and clinical infections.

Results The study revealed substantial asymptomatic infections with average parasite carriage of $8.11 \%$ and $7 \%$ in Dielmo and Ndiop, respectively. P. falciparum accounted for most asymptomatic infections (more than 90\%). In Dielmo, 95\% of asymptomatic infections clustered within the same geographical areas while infections were disparate in Ndiop. Preliminary fine-scale mapping of asymptomatic and clinical malaria infections identified clusters of higher malaria incidence interpreted as foci of transmission across the four-year study period with 95\%-98\% of clinical infections occurring in households where an asymptomatic malaria infection existed.

Conclusion This study revealed substantial asymptomatic Plasmodium infections in both settings throughout the four-year study period and spatial clusters of malaria infections at the microepidemiological level. Together, these findings could offer a feasible approach for a rational targeting of malaria control interventions to achieve elimination.

\section{PO 8421 LITERATURE REVIEW OF BIOMARKERS FOR HUMAN AFRICAN TRYPANOSOMIASIS POST-TREATMENT FOLLOW-UP}

${ }^{1}$ Lukusa Ngay*, ${ }^{2}$ Veerle Lejon, ${ }^{1}$ Mumba Ngoyi. ${ }^{1}$ Institut National de Recherche Biomedicale (INRB), Kinshasa, Democratic Republic of the Congo; ${ }^{2}$ Institut de Recherche pour le developpement (IRD)/Montpellier, France

\subsection{6/bmjgh-2019-EDC.92}

Introduction Human African trypanosomiasis (HAT) is caused by Trypanosoma brucei gambiense and rhodesiense and is transmitted to humans by tsetse flies in sub-Saharan Africa. To detect cure or treatment failure, patients are followed up after treatment integrating the use of biomarkers in blood or cerebrospinal fluid (CSF).

Methods A systematic review of the literature according to the PRISMA Statement for Reporting Systematic Reviews was done, focusing on biological markers for HAT post-treatment follow-up. Articles were retrieved from PubMed (https://www. ncbi.nlm.nih.gov/pubmed/) by using keywords: Human African Trypanosomiasis, Biomarkers, Follow up, Post treatment.

Results A panel of biomarkers is used to detect relapses or to confirm recovery. For post-treatment follow-up, an examination of the CSF is performed. White blood cell counts in CSF with a defined cut-off value have been proven to be the most accurate to assess the treatment outcome. The intrathecal immunoglobulin $M$ synthesis is a specific and sensitive parameter for 
the detection of CNS involvement in cases of HAT caused by T. brucei gambiense. The decrease of trypanosome-specific antibodies concentrations in CSF could be a good parameter for definite cure. High CSF IL-10 levels during treatment followup indicate recurring CNS inflammation and treatment failure. An increase of Neopterin in CSF and the presence of trypanosome spliced leader RNA in the blood have a high potential as predictors for treatment failure but need further validation.

Conclusion New biomarkers for post-treatment follow-up in HAT should 1) have high diagnostic specificity and sensitivity; 2) be applicable in field conditions; 3) preferentially be performed on blood and thus avoid the painful lumbar puncture during post-treatment control visits; and 4) shorten the follow-up period.

\section{PO 8425 THE DIAGNOSTIC AND PROGNOSTIC VALUE OF NEW URINE-BASED LEISHMANIA ANTIGEN DETECTION TESTS}

Salah Boshara. Institute of Endemic Diseases, University of Khartoum, Sudan

\subsection{6/bmjgh-2019-EDC.93}

Background Visceral leishmaniasis (VL) also known as kala-azar, is a protozoan infection caused by the L. donovani complex and transmitted by sandflies. Early detection of leishmaniasis is critical in management of patients and for successful control and elimination of the disease. Definitive diagnosis of visceral leishmaniasis is by parasitological demonstration of parasites in splenic, lymph node or bone marrow aspirates, which are collected using invasive methods that are unsuitable in the field. This study aimed to evaluate new less invasive urine-based ELISA and rapid diagnostic test (RDT) assays for diagnosis of VL.

Methods The newly developed urine ELISA test was evaluated using archived and fresh urine samples collected from parasitologically confirmed VL patients and non-VL cases. Lateral flow assay (LFA) using the ELISA reagents were conducted for day0 samples. Serological tests (DAT, rk28 ICT) were conducted for every patient in the study.

Results In 198 patients with suspected VL, urine rapid test had a sensitivity of $72.2 \%$ and exhibited a specificity of 93.42\%. Leishmania antigen ELISA had a sensitivity of $83.33 \%$ and a specificity of $95.05 \%$. All VL-confirmed cases were followed up during the treatment period, the Leishmania antigen ELISA became negative 2 months after completion of treatment in most patients.

Conclusion Urine lateral flow assay is a simple addition to the diagnostics of VL particularly at field level and as a complementary test for the diagnosis of VL in smear-negative cases. Further enhancement of the test will define its performance in monitoring treatment. Further studies are recommended to evaluate the performance of both tests in the diagnosis of HIV-co-infected cases.

\section{PO 8426 DELIVERY OF POST-DISCHARGE MALARIA CHEMOPREVENTION (PMC) WITH DIHYDROARTEMESININ-PIPERAQUINE FOR MANAGEMENT OF CHILDREN WITH SEVERE ANAEMIA IN MALAWI}

${ }^{1}$ Thandile Gondwe, ${ }^{2}$ Bjarne Robberstad, ${ }^{1}$ Mavuto Mukaka, ${ }^{2}$ Bjorn Blomberg, ${ }^{1}$ Kamija Phiri. ${ }^{1}$ University of Malawi, Zomba, Malawi; ${ }^{2}$ University of Bergen, Norway

10.1136/bmjgh-2019-EDC.94
Background Children hospitalised with severe anaemia in Africa are at high risk of readmission or death within 6 months after discharge. No strategy addresses this post-discharge period. In Malawi, 3 months of post-discharge malaria chemoprevention (PMC) with monthly 3 day courses of artemether-lumefantrine (AL) in children with severe malarial anaemia prevented $31 \%$ of deaths and readmissions. There is now a need to design and evaluate an effective delivery strategy for PMC.

Methods This is a cluster-randomised trial whose primary objective is to determine the optimum PMC delivery strategy by comparing community versus health facility-based strategies with the aim to inform policy. Convalescent children under 5 years old, weighing $>5 \mathrm{~kg}$, admitted with severe anaemia and clinically stable are included. All children received dihydroartemisinin-piperaquine 2, 6 and 10 weeks after discharge, either: 1) at discharge with SMS reminder; 2) at discharge without an SMS reminder; 3) at discharge and community health worker reminder; 4) at the hospital with an SMS reminder; or 5) at the hospital without an SMS reminder. The primary outcome measure is uptake of courses of PMC drugs. Children will be followed up for 15 weeks. The sample size is 75 children per arm (375 total).

Results The study has nearly completed enrollment and preliminary data analysis is in progress. We expect to identify the most effective, cost-effective, acceptable and feasible strategy for delivering intermittent preventive therapy post-discharge for management of severe anaemia in under-five children.

Conclusion The findings of this study will be presented; they address the knowledge gap regarding the potentially preventable component of the burden that occurs after discharge from hospital, and inform the optimal delivery strategy for PMC.

\section{PO 8430 A GEOSPATIAL APPROACH TO PREDICTING DIARRHEA PREVALENCE IN NIGERIA}

Oluwasegun Adetunde. Department of Geography and Environmental Management, University of Ilorin, Nigeria

\subsection{6/bmjgh-2019-EDC.95}

Background Nigeria ranks second globally only behind India in under-five mortality prevalence. In Nigeria, 108.8 children die per 1000 live births before their 5th birthday. It is of note that diarrhoea $(15.3 \%$ prevalence $)$ is the second leading cause of under-five mortality in Nigeria after pneumonia. General poor hygiene and nutritional status are contributory factors to diarrhea.

Methods Data was collected for severe acute malnutrition (SAM) using the weight for height z-value (WHZ) and/or oedema criteria. In addition, data on diarrhoea prevalence, oral rehydration salt therapy (ORST), improved source of drinking water and improved sanitation were collected. These were obtained for 36 states and federal capital territory (FCT) from the National Bureau of Statistics headquarters in FCT, Abuja for 2015. Correlation analysis was first carried out to determine relationships followed by geographically weighted regression analysis (GWR). GWR was used to predict underfive mortality pattern and accuracy mapped.

Results Observed correlation coefficients to diarrhoea prevalence were $0.59,-0.49,-0.35$ and -0.63 for SAM, ORST, improved drinking water access, and improved sanitation, 